\title{
Application of New Surveying and Mapping Technology in the Construction of Smart City
}

\author{
Xiaoping $\mathrm{Shi}^{*}$, Bin Wang ${ }^{2}$ \\ ${ }^{1}$ School of Civil and Transportation Engineering, Shanghai Urban Construction Vocational College, Shanghai, 200438, China \\ ${ }^{2}$ Geotechnical Department, Shanghai Harbour Engineering Quality Inspection Co., Ltd, Shanghai, 200032, China
}

\begin{abstract}
With the rapid development of information technology, the rapid advancement of urbanization and the rapid expansion of urban scale, higher requirements have been put forward for the development of the city, which promotes the continuous acceleration of the urban process, and the construction of smart city has become an inevitable development trend. Smart city is the combination of digital city and Internet of things. Digital city construction uses Remote Sensing, Global Navigation Satellite System, Geographic Information System, Engineering Survey and other Mapping Technology. Therefore, in the construction of smart city, the most indispensable is modern information technology and surveying and mapping technology. Through the analysis of the application of new surveying and mapping technology in the construction of smart city, this paper shows the important role of new surveying and mapping technology in the construction of smart city.
\end{abstract}

\section{Introduction}

Urban construction is a subjective activity that people consciously transform the natural environment and arrange the urban architectural space and material environment in the process of urban development. With the development of social and economy, people's requirements for the quality of life are constantly improving, and the construction of the city is changing with each passing day. Urban construction has developed from traditional city construction to digital city construction, and digital city construction to smart city construction. The combination of New Generation Internet, Cloud Computing, Intelligent Sensing, Communication and other Internet of things technologies with Remote Sensing, Global Navigation Satellite System, Geographic Information System and other surveying and mapping technologies can realize intelligent identification, positioning, tracking, monitoring and management of all objects, thus making the construction of smart city technically possible. Therefore, this paper expounds the application of Surveying and mapping technology in the construction of smart city, as follows.

\section{Application of new surveying and mapping technology in smart city construction}

\subsection{The importance of new surveying and mapping technology in the construction of smart city}

Surveying and mapping refers to the measurement, collection and mapping of the shape, size, spatial position and attributes of natural geographical elements or artificial facilities on the surface. New modern surveying and mapping technologies, such as Global Navigation Satellite System, Remote Sensing, Geographic Information System, Surveying Robot, Photographic surveying and measurement technology of 3D laser scanning, are combined with cloud computing processing technology and Internet of things technology. It provides basic data and application support for the construction of smart city, making the city construction more and more intelligent.

\subsection{New surveying and mapping technology provides basic surveying and mapping geographic information for smart city construction}

Basic surveying and mapping geographic information mainly includes data, graphics, images and other achievements and derivatives. Premier Keqiang Li once said: The development of national economy is inseparable 
from surveying and mapping geographic information, and the national modernization construction is even more inseparable from surveying and mapping geographic information. It is impossible to build a smart city without surveying and mapping geographic information, which is the basis of smart city construction. Generally, the construction of the Internet of things in smart city needs a lot of urban surveying and mapping data. Urban mapping data mainly includes two kinds, namely geographic data and comprehensive management data. Among them, geographic data is mainly realized by surveying and mapping technology, which can effectively ensure the security of geographic data and make better use of urban geographic data. With the development of science and technology in China, the surveying and mapping technology in the past is complicated, the precision is poor, and the working efficiency is slow. Therefore, combining with the development of modern technology, our country constantly studies new surveying and mapping technology. The new surveying and mapping technology is based on computing technology and network to realizes the intelligent measurement instrument. The new technology provides more timely, accurate and rapid geospatial information for the construction of smart city.

\subsubsection{Provide basic spatial data.}

With the rapid development of science and technology, Measuring Robots, Digital Level, GNSS and threedimensional laser scanner and other high-precision and intelligent measuring instruments have emerged, which provide accurate and reliable basic spatial data for the construction of smart city.

(1) Measuring Robot.

The measuring robot is a kind of intelligent total station, which integrates automatic target recognition, automatic collimation, automatic angle measurement and ranging, automatic target tracking and automatic recording. With the application of optoelectronic technology, computer technology and other new technologies in total station, the total station gradually develops towards automation and intelligence. These new technologies provide hardware basis for improving measurement accuracy, reducing labor intensity, working in special environment and expanding work field. At present, the measurement accuracy of measuring robot can reach $0.5 "$ in the angle measurement accuracy, and the ranging accuracy is $0.6 \mathrm{~mm}+1 \mathrm{ppm}$. It integrates ultra-high precision total station technology, highresolution digital image technology and super mirror station technology. Ultra-high precision total station can meet the diversified needs of the current measurement industry. It provide more accurate and reliable basic spatial data for smart city construction.

(2) Digital Level.

The digital level has the following advantages: the reading is objective, there is no human error and error recording; the accuracy is high, the accuracy can reach $0.3 \mathrm{~mm} / \mathrm{km}$; the speed is fast, the measurement time is reduced about $1 / 3$ of the time compared with the traditional level; the efficiency is high, only need to focus and press the measurement key to complete the automatic measurement. The survey data has high precision and provides terrain elevation data for generating high-quality digital elevation model. Digital elevation model is widely used in surveying and mapping, hydrology, meteorology, landform, geology, soil, engineering construction, communication, military and other national economy and national defense construction, as well as humanities and natural science fields. To meet the needs of intelligent construction of smart city.

(3) Global Navigation Satellite System (GNSS).

GNSS is mainly transmitted to the ground receiver through the signal by artificial satellite. The receiver can accurately grasp the measurement position and specific position information, so as to achieve good measurement effect. The method of simultaneous determination of three-dimensional coordinates by GNSS extends mapping and positioning technology from land and offshore to the whole ocean and outer space, from static to dynamic, from post-processing to real-time positioning and navigation. GNSS is the infrastructure of national security and economy, and an important symbol of modern power status and comprehensive national strength. With the continuous development of GNSS technology and the improvement of Beidou system, the measurement level has been improved and the measurement is more comprehensive and reliable. GNSS will not be interfered by weather factors, terrain factors and external conditions. It can complete the measurement work efficiently and accurately, and will not produce measurement errors by influence of external factors. It can effectively get rid of the measurement difficulties caused by terrain factors and environmental factors.

(4) Measurement technology of 3D laser scanning.

Measurement technology of 3D laser scanning is called "real scene replication technology", which is another technological revolution in surveying and mapping field after GPS technology. By using the method of laser beam high-speed scanning measurement, it can quickly obtain the $3 \mathrm{D}$ coordinate data of each sampling point on the surface of the measured object in a noncontact way. It can quickly, large area and high-resolution collect spatial point information, and obtain a collection of solid points. The development of terrain survey has promoted the development of topographic survey to hightech three-dimensional graphics.

3D laser scanning can be widely used in the following aspects: (1) 3D modeling of buildings and structures, such as houses, pavilions, temples, towers, castles, churches, bridges, viaducts, overpasses, roads, offshore oil platforms, refinery pipelines, etc; (2) small scale digital terrain models or elevation models, such as golf course, motorcycle steeplechase racetrack, rock wall, etc; (3) 3D models of independent objects, such as airplanes, ships, automobiles, statues, etc; (4) Three dimensional models of natural landforms, such as caves. For example, with the continuous development of society, people's awareness of environmental protection is also higher and higher. With the implementation of sustainable development strategy, the construction of digital mine is of great significance to the protection of ecological environment. With the increasing of mining in China, the ecological environment 
has been seriously damaged. Through the 3D laser scanning, the mine information is measured, and the threedimensional model of the mine is established to analyze the more seriously damaged areas. Some measures are taken to improve the mine ecosystem, avoid the occurrence of disasters. It reflect the connotation of human-oriented and sustainable development of smart city, and make intelligent response to the environmental protection and public safety requirements of smart city, and creates better urban life for human beings.

\subsubsection{Provide sensing image data.}

Under the background of the rapid development of modern science and technology, the new measurement technology based on the application of Digital Aerial Photogrammetry, Low Altitude Photogrammetry, Tilt Photogrammetry and High-resolution Satellite Remote Sensing has been widely used in the construction of smart city. It can not only obtain the measurement information needed for the construction of smart city, but also control the smart city by high precision image acquisition, and create conditions for visualization technology of urban geographic information. At the same time, the surveying and mapping information can be compared, and the change of urban development can be predicted more accurately.

(1) Digital Aerial Photogrammetry.

Digital Aerial Photogrammetry is an important method of large area and large scale topographic mapping and cadastral survey. It can provide various forms of map products, such as digital, image and line drawing. With the application of digital aerial photogrammetry, photogrammetry products are transformed from image map to 4D (DSM, DEM, DOM and DLG), which provides reliable data guarantee for basic geographic information platform. Aerial images can update and analyze the land and resources survey and land use capability in urban management, and help to grasp the situation of urban land resources utilization more quickly. It is helpful to improve the urban environment to grasp the changes of relevant environmental factors in urban areas through aerial images, and to know whether there is pollution in land, water and vegetation in real time. Through aerial photogrammetry, we can explicit the land texture information and landscape vegetation information in different areas of the city, and provide more information for urban landscape engineering according to the monitoring of landscape plants. The use of aerial photogrammetry technology can continuously improve the level of urban management and realize the smart development of the city.

(2) Low Altitude Photogrammetry.

Low Altitude Photogrammetry is a kind of digital aerial Photogrammetry technology with altitude below $1000 \mathrm{~m}$. It mainly uses low altitude UAV for photogrammetry, which has the incomparable mobility and flexibility, and can perform rapid mapping tasks under complex ground and meteorological conditions. Low altitude photogrammetry is mainly used in disaster emergency mapping, small-scale and large-scale topographic map mapping, three-dimensional city modeling and cultural relics Archaeology and protection. In the process of smart city construction, the highresolution low altitude image can be used as the image source of city $3 \mathrm{D}$ model, so as to give full play to the multiple functions of image data. It plays an important role in smart city management, smart tourism, smart public security and so on.

(3) Tilt Photogrammetry.

Tilt Photogrammetry technology is a high-tech developed in the field of international Photogrammetry technology in recent ten years. It can obtain rich highresolution texture of building top and side view by synchronously collecting images from one vertical, four inclined and five different perspectives. It can not only reflect the real situation of the ground objects and obtain the texture information of the objects with high precision, but also generate the real three-dimensional city model through the advanced positioning, fusion, modeling and other technologies. The technology can be used in emergency command, land security, urban management and other industries.

(4) High Resolution Satellite Remote Sensing.

At present, Remote Sensing has been applied and developed in many fields, such as mapping of topographic map, investigating and monitoring of land and resources, providing effective services for disaster prevention and mitigation, agriculture, forestry and water conservancy, ecological environment, urban planning and construction, transportation and national defense construction. The combination of remote sensing technology, GNSS technology and geographic information technology can reasonably plan urban traffic lines and agricultural production. It provide data basis and conditions for smart city construction.

(5) Lidar System.

The lidar system can not only obtain 3D ground geographic information, but also directly obtain low-cost, high-density, high-speed and high-precision digital elevation data or digital surface data. The obtained data can be widely used in resource exploration, urban planning, agricultural development, water conservancy engineering, land use, environmental monitoring, traffic communication, earthquake prevention and disaster reduction and national key points construction projects, etc. It provides extremely important raw materials for national economy, social development and scientific research, and makes an important guarantee for the multidimensional construction of smart city.

\subsection{New surveying and mapping technology to build a smart city space-time information cloud platform}

Smart city takes spatial location as the connection point, and integrates various data resources with geographic information system and virtual reality technology. Geographic Information System (GIS), which can analyze and process the spatial information, is a computer system for inputting, storing, querying, analyzing and displaying geographic data. It is a new technology gradually 
developed in recent years. It uses computer terminal to accurately carry out geographic positioning and data dynamic analysis, and finally express spatial information in the form of graphics or data. The main content of smart city construction is to connect urban construction, management, economy, people's livelihood and other information with spatial geographic location information by using spatial geographic information system. In order to meet the requirements of smart city development, it is necessary to build a spatiotemporal information cloud platform based on big data. On the platform of GIS, cloud computing is used in the modeling, storage and processing of GIS to realize data sharing, exchange, integration and access, etc. It provides data for urban management, civil affairs, transportation, industry and commerce, planning, municipal garden, environmental protection, public safety, communication, first aid, electric power, water conservancy, facility management and resource management and make intelligent decisions, so as to make urban operation, management and service more efficient, more intelligent, more flexible and more convenient.

\section{Conclusion}

The construction of smart city needs a large amount of data to support its intelligent functions. These data can be obtained through surveying and mapping technology, which provides basic information and technical support for the construction of smart city. Therefore, we must attach importance to the development of Surveying and mapping technology. We should closely combine the development of modern high-tech and the needs of actual production, and form advanced and reliable new surveying and mapping technology through improving and innovating surveying and mapping technology, so as to lay a good foundation for the construction of smart city and promote the stable and rapid development of China's smart city.

\section{Acknowledgments}

Thank you very much for my colleagues of surveying and mapping major in school. They have provided me a lot of help and encourage.

\section{References}

1. Li,C.M., Liu,X.L., Yin,J. (2013) Thinking and exploration of mathematical city to smart city. Surveying and mapping bulletin, 000: 1-3.

2. Li,D., Yao,Y., Shao,Z.F. (2012) Concept, supporting technology and application of "smart city". Engineering research, 000: 11-17.

3. Zhang,W. (2014) Application of key technologies in smart city construction. Chang'an University, Xi'an.

4. Wu,H. (2017) Application of new surveying and mapping technology in smart city construction . Smart city, 003: 17-19.
5. Jiang,W.M. (2019) The role of Surveying and mapping technology in the construction of smart city . Metallurgy and materials, 039: 77-78.

6. Liu,K., Gao,C.1. (2018) Application of new surveying and mapping technology in smart city construction. Intelligent building and smart city, 046: 111-112. 